\title{
Tonsillar Crypt
}

National Cancer Institute

\section{Source}

National Cancer Institute. Tonsillar Crypt. NCI Thesaurus. Code C33791.

One of many deep mucosal invag inations on the medial surface of the palatine tonsil that extend through the whole thickness of the tonsil, which serve to increase the surface area of the tonsil. 